\title{
An unpleasant tenant - a conundrum in an emergency
}

\author{
Abraham Paul ${ }^{1}$ \\ ${ }^{1}$ Apollo Adlux Hospital
}

February 22, 2022

\begin{abstract}
A 60yr old hypertensive patient presented with inferior wall myocardial infarction. His coronary angiogram showed ectatic coronaries, with a large thrombus in an aneurysmally dilated right coronary artery. Emergency coronary artery bypass grafting was performed, and he was discharged on the seventh post operative day.
\end{abstract}

\section{An unpleasant tenant - a conundrum in an emergency}

Key words - intracoronary thrombus, aneurismal coronary artery, inferior wall myocardial infarction History

A $60 y$ r old male, hypertensive, presented with chest pain. ECG showed inferior wall myocardial infarction with complete heart block, corroborated by echocardiography which also showed right ventricular dysfunction. He was taken up for Primary PTCA under temporary pacing.

CAG showed ectatic left sided vessels with slow flow (figure 1,2). 


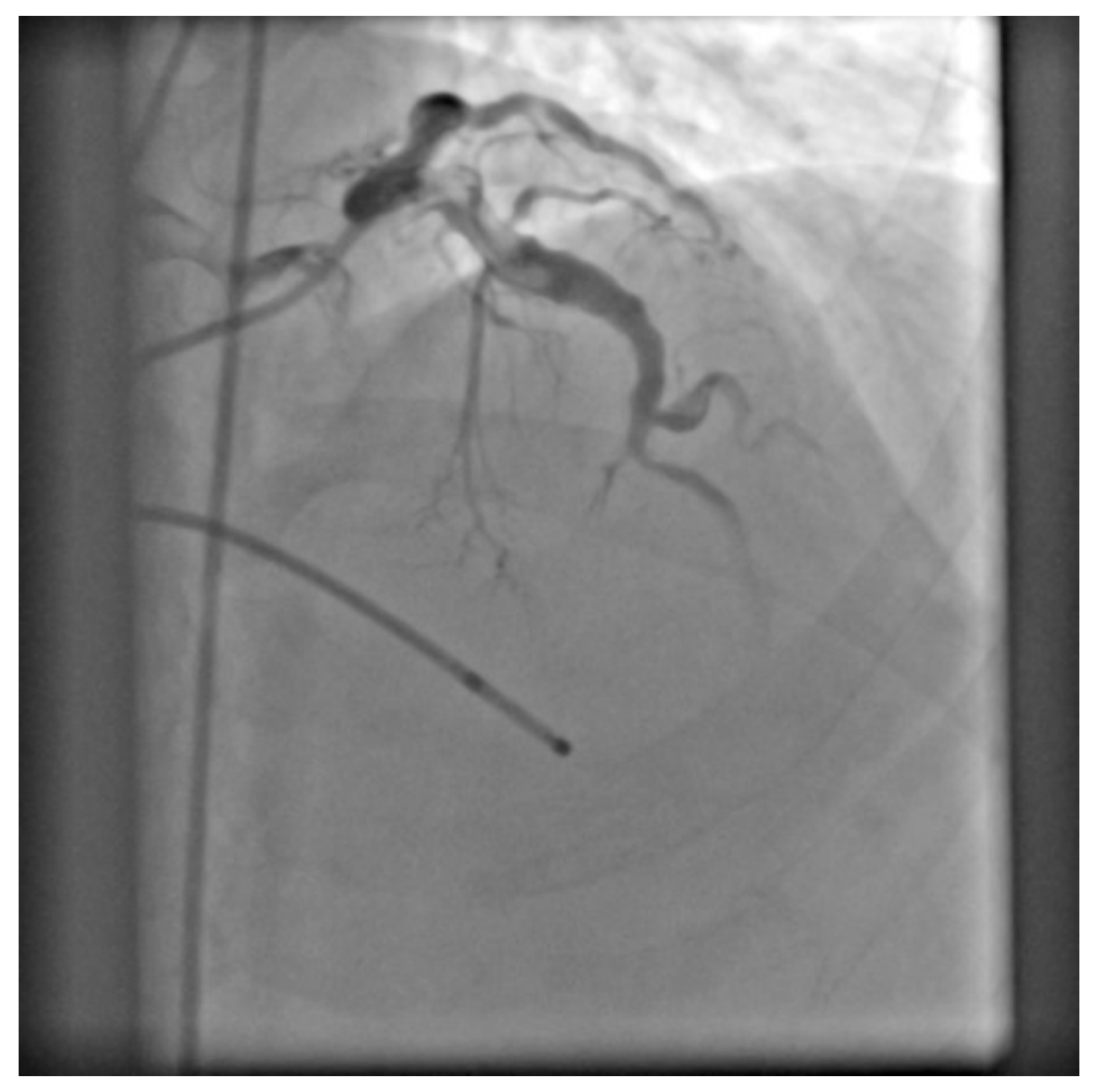

Figure 1. ectatic Left Anterior Descending Artery 


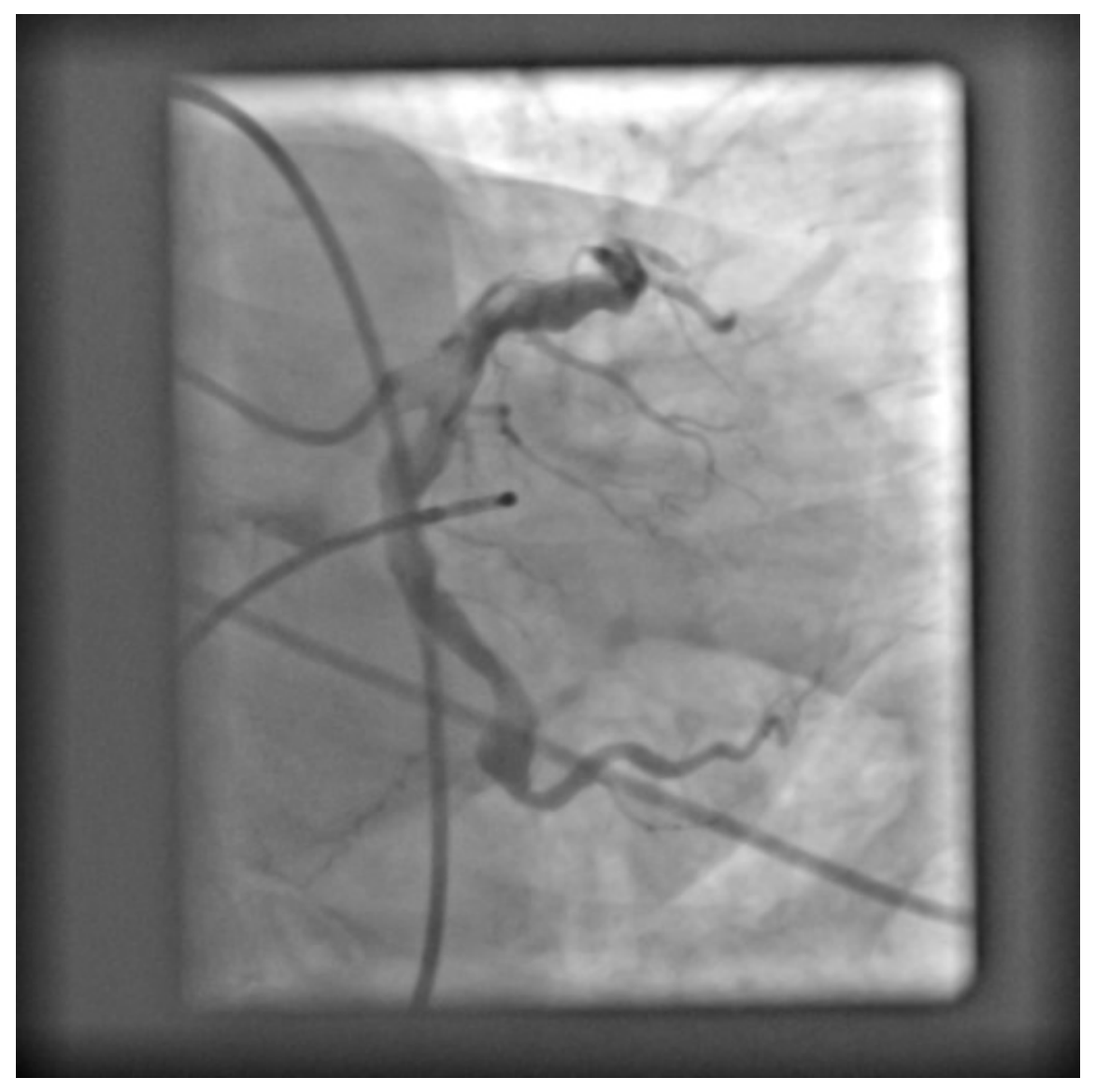

Figure 2. Ectatic Left Circumflex Artery

Right coronary injection revealed a large proximal aneurysm with organized thrombus completely occluding the vessel. 


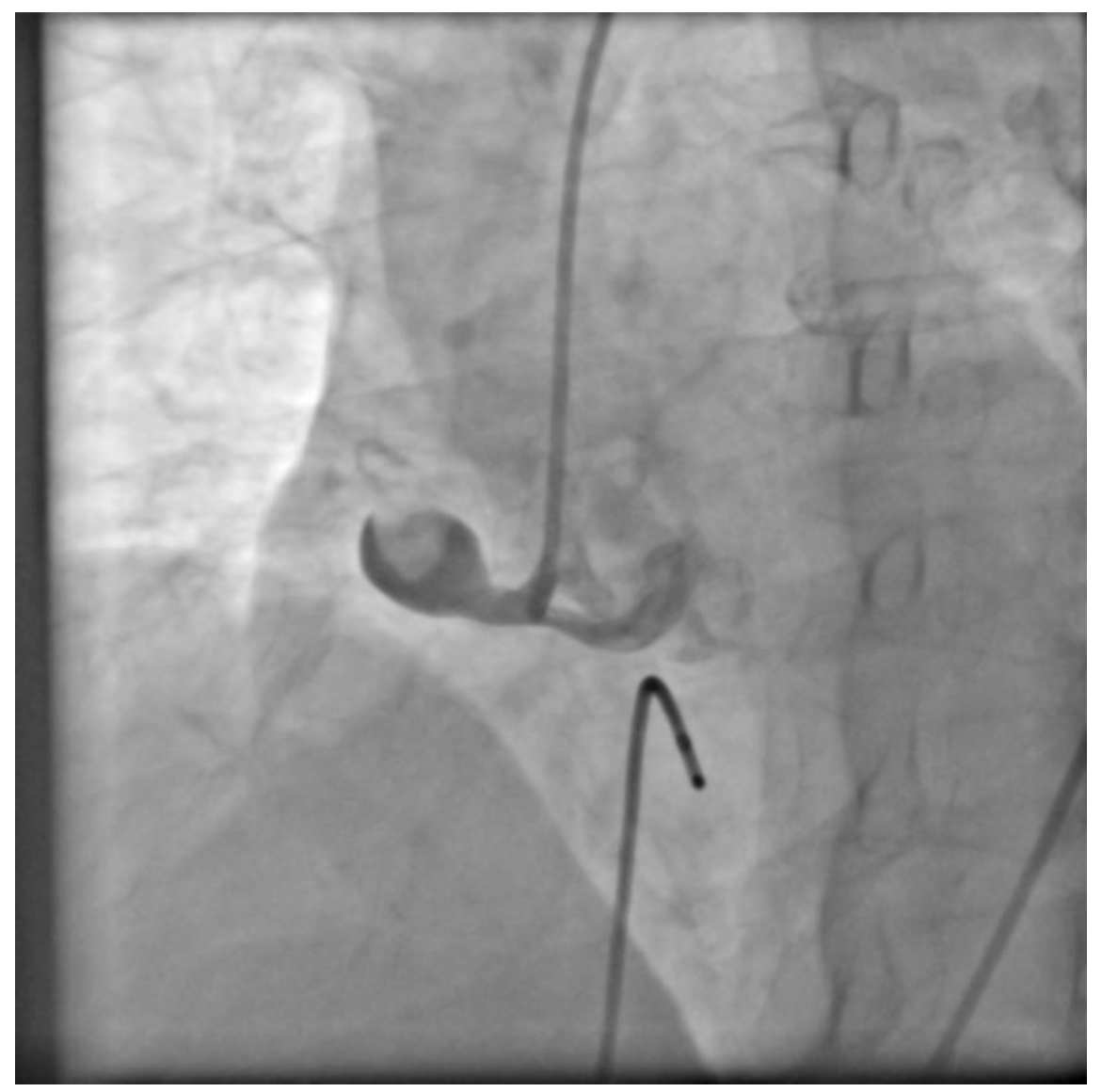

Figure . Right Coronary Artery injection showing organized thrombus in proximal aneurysm

Attempts were made to cross the occluded segment, however aggressive manipulation was deferred due to fear of proximal dislodgement and subsequent cerebral embolism. He developed ventricular fibrillation thrice inside the cath lab, which was reverted with DC shock. He was taken up for emergency Coronary Artery Bypass Grafting. Post surgery he was noted to have left hemiparesis, and a CT brain confirmed right frontal lobe acute infarct. He maintained sinus rhythm after surgery, and was discharged 7 days after index event, on dual anitplatelets and warfarin.

\section{Discussion}

Coronary artery aneurysm is a rare disorder, which occurs in $0.3 \%-4.9 \%$ of patients undergoing coronary angiography ${ }^{1}$. Atherosclerosis accounts for $>90 \%$ of coronary artery aneurysms in adults, whereas Kawasaki disease is responsible for most cases in children ${ }^{2}$. With the advent of implantation of drug-eluting stents, there are increasing reports suggesting stents causing coronary aneurysms, months or years after the procedure ${ }^{3}$.

Optimal management strategy has to be individualized using a combination of the size of the aneurysm, expansion history, pathophysiology, and symptoms to decide on intervention. The available percutaneous options include conventional stent implantation, coil embolization, autologous saphenous vein-covered stent grafting, and polytetrafluoroethylene (PTFE)-covered stent graft ${ }^{4}$. Surgical strategies including resection, aneurysm ligation, marsupialization with interposition graft, and coronary artery bypass surgery.

The indications for the surgical treatment of CAA in general are:

- Severe coronary artery disease 
- CAAs near the bifurcation of large branches

- Evidence of emboli from the aneurysm to the distal coronary bed resulting in myocardial ischemia

- Progressive enlargement of a CAA documented by serial angiographic measurements; and

- CAAs in the LMS

- Complications such as fistula formation

- Compression of cardiac chambers

- Giant CAA (dilatation exceeding the reference vessel diameter by $>$ four times).

The prognosis of CAA depends on the size of the aneurysm. In general, small aneurysms have a favorable prognosis with a low risk of myocardial ischemic events and/or mortality. On the contrary, giant CAAs (i.e., those with an internal diameter $>8 \mathrm{~mm}$ ) have a high risk of morbidity and mortality. About one-half of such aneurysms become obstructed, and are associated with myocardial infarction, arrhythmias, or sudden death.

\section{Authors contribution}

Dr Abraham Paul, corresponding author

\section{References}

1. Aneurysmal coronary artery disease. Swaye PS, Fisher LD, Litwin P, Vignola PA, Judkins $M P$, Kemp $H G$, Mudd JG, Gosselin AJ Circulation. 1983 Jan; 67(1):134-8.

2. Coronary artery aneurysms and ectasia: role of coronary CT angiography. Díaz-Zamudio M, BacilioPérez U, Herrera-Zarza MC, Meave-González A, Alexanderson-Rosas E, Zambrana-Balta GF, KimuraHayama ET Radiographics. 2009 Nov; 29(7):1939-54.

3. Coronary artery aneurysms after drug-eluting stent implantation. Aoki J, Kirtane A, Leon MB, Dangas G JACC Cardiovasc Interv. 2008 Feb; 1(1):14-21.

4. Use of polytetrafluoroethylene-covered stent for treatment of coronary artery aneurysm. Szalat A, Durst $R$, Cohen A, Lotan C Catheter Cardiovasc Interv. 2005 Oct; 66(2):203-8. 


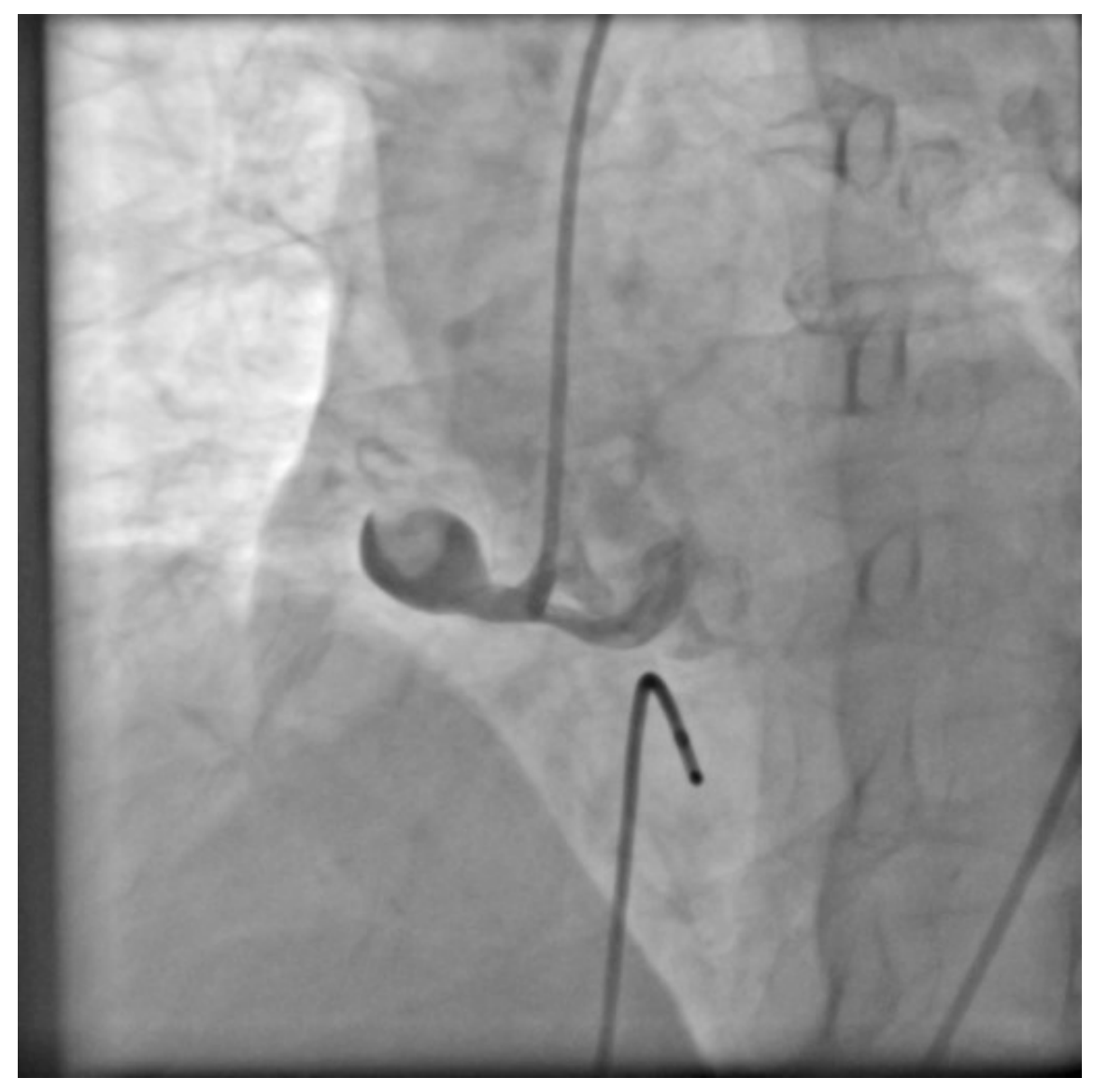

